\title{
A local-global criterion for dynamics on $\mathbb{P}^{1}$
}

\author{
by \\ Joseph H. Silverman (Providence, RI) and \\ José Felipe Voloch (Austin, TX)
}

Introduction. A recent paper of Hsia and Silverman [3] describes an analog of the Brauer-Manin obstruction in the setting of arithmetic dynamics. The definition is based on work of Scharaschkin [6], who showed how to reformulate the Brauer-Manin obstruction on curves of genus at least 2 as a purely adelic-geometric statement, with no reference to cohomology. (See also [5].) In this note we prove a dynamical analog of Scharaschkin's conjecture for dynamical systems on $\mathbb{P}^{1}$. The proof uses a variety of tools, including a Zsigmondy theorem for primitive divisors in dynamical systems that was recently proven by Ingram and Silverman [4].

We recall from [3] the setup for the dynamical version of the BrauerManin obstruction.

Notation. Let $K$ be a number field, let $X / K$ be a projective variety, and let $\varphi: X \rightarrow X$ be a $K$-morphism of infinite order. Let $\mathrm{A}_{K}$ denote the ring of adeles of $K$, and for any point $P \in X(K)$, write $\mathrm{C}\left(\mathcal{O}_{\varphi}(P)\right)$ for the closure of the orbit $\mathcal{O}_{\varphi}(P)$ of $P$ in $X\left(\mathrm{~A}_{K}\right)$. Let $Z$ be a subvariety of $X$. We clearly have an inclusion

$$
\mathcal{O}_{\varphi}(P) \cap Z(K) \subset \mathrm{C}\left(\mathcal{O}_{\varphi}(P)\right) \cap Z\left(\mathrm{~A}_{K}\right) .
$$

The primary question raised in [3] is to determine when the inclusion (1) is an equality.

More generally, we can ask a similar question after excluding a (finite) set of places. For any set of places $S$ we let

$$
\mathrm{A}_{K, S}^{\prime}=\prod_{v \notin S}^{\prime} K_{v} \subset \mathrm{A}_{K},
$$

2000 Mathematics Subject Classification: Primary 11B37; Secondary 11G99, 14G99, 37F10.

Key words and phrases: arithmetic dynamical systems, local-global criterion, BrauerManin obstruction. 
where the product is the usual adelic restricted product, and we write $\mathrm{C}_{S}\left(\mathcal{O}_{\varphi}(P)\right)$ for the closure of the orbit in $\mathrm{A}_{K, S}^{\prime}$.

Our main result is a proof of a local-global equality (1) for $X=\mathbb{P}^{1}$.

TheOREM 1. Let $K$ be a number field, let $S$ be a finite set of places of $K$, and let $\varphi: \mathbb{P}^{1} \rightarrow \mathbb{P}^{1}$ be a rational map of degree at least two defined over $K$. Let $Z \subset \mathbb{P}^{1}(K)$ be a finite set of points. Then with notation as above,

$$
\mathcal{O}_{\varphi}(P) \cap Z(K)=\mathrm{C}_{S}\left(\mathcal{O}_{\varphi}(P)\right) \cap Z\left(\mathrm{~A}_{K, S}^{\prime}\right) .
$$

REMARK 2. As noted in [3], if (1) is to be an equality, one should require that the subvariety $Z$ contains no positive-dimensional $\varphi$-preperiodic subvarieties. However, since our subvariety $Z$ is a set of points, this condition on $Z$ is vacuous.

In Section 2 we prove an analogous result for function fields $K / k$. If the map $\varphi$ is not isotrivial, the proof is essentially the same as the proof of Theorem 1, but the isotrivial case must be handled separately. Finally, in Section 3, we make some further comments and raise some related questions.

1. Local-global dynamics on $\mathbb{P}^{1}$ over number fields. In this section we prove Theorem 1 . We briefly recall some basic definitions from dynamical systems.

Definition. Let $\varphi(z) \in K(z)$ be a rational function of degree $d \geq 2$, which we may view as a morphism $\varphi: \mathbb{P}_{K}^{1} \rightarrow \mathbb{P}_{K}^{1}$. A point $\gamma \in \mathbb{P}^{1}(\bar{K})$ is periodic for $\varphi$ if $\varphi^{n}(\gamma)=\gamma$ for some $n \geq 1$. The smallest such $n$ is called the $\varphi$-period of $\gamma$. We say that $\gamma$ is preperiodic for $\varphi$ if its $\varphi$-orbit

$$
\mathcal{O}_{\varphi}(\gamma)=\left\{\gamma, \varphi(\gamma), \varphi^{2}(\gamma), \ldots\right\}
$$

is finite. Equivalently, $\gamma$ is preperiodic if some iterate $\varphi^{n}(\gamma)$ is periodic.

REMARK 3. During the proof of Theorem 1, we make use of the following notation. For any two points $x, y \in \mathbb{P}^{1}(K)$ and any prime ideal $\mathfrak{p}$ of $K$, we write

$$
x \equiv y(\bmod \mathfrak{p})
$$

to indicate that the reductions of $x$ and $y$ modulo $\mathfrak{p}$ coincide. Formally, this notation means that if we write $x=\left[x_{1}, x_{2}\right]$ and $y=\left[y_{1}, y_{2}\right]$ using $\mathfrak{p}$-integral coordinates such that at least one coordinate is a $\mathfrak{p}$-unit, then

$$
x_{1} y_{2}-x_{2} y_{1} \equiv 0(\bmod \mathfrak{p}) .
$$

Alternatively, define a (nonarchimedean) chordal metric on $\mathbb{P}^{1}$ for the absolute value $v$ by (cf. $[11, \S 2.1]$ )

$$
\Delta_{v}(x, y)=\frac{\left|x_{1} y_{2}-x_{2} y_{1}\right|_{v}}{\max \left\{\left|x_{1}\right|_{v},\left|x_{2}\right|_{v}\right\} \cdot \max \left\{\left|y_{1}\right|_{v},\left|y_{2}\right|_{v}\right\}} .
$$


The value of $\Delta_{v}(x, y)$ is independent of the choice of homogeneous coordinates for $x$ and $y$, and we define the congruence (2) to mean $\Delta_{v}(x, y)<1$, where $v$ is the absolute value associated to the prime $\mathfrak{p}$. More generally, for points $\alpha_{i}, \beta_{i} \in \mathbb{P}^{1}(K)$, we write

$$
\prod\left(\alpha_{i}-\beta_{i}\right) \equiv 0(\bmod \mathfrak{p})
$$

to mean that $\prod \Delta_{v}\left(\alpha_{i}, \beta_{i}\right)<1$, in which case we say that $\prod\left(\alpha_{i}-\beta_{i}\right)$ is divisible by $\mathfrak{p}$. Equivalently, (3) means that there is some index $j$ such that $\alpha_{j}$ and $\beta_{j}$ have the same reduction modulo $\mathfrak{p}$.

REMARK 4 . We recall that there are several equivalent definitions for the map $\varphi: \mathbb{P}_{K}^{1} \rightarrow \mathbb{P}_{K}^{1}$ to have good reduction at $\mathfrak{p}$; see [11, Theorem 2.5]. For example, $\varphi$ has good reduction if it can be written as a ratio of polynomials $\varphi=F / G$ such that $F$ and $G$ have $\mathfrak{p}$-integral coefficients and such that the resultant $\operatorname{Res}(F, G)$ is a p-adic unit. It is clear that $\varphi$ has good reduction at all but finitely many primes of $K$. Further, if $\mathfrak{p}$ is a prime of good reduction associated to the absolute value $v$, then [11, Theorem 2.17] states that

$$
\Delta_{v}(\varphi(x), \varphi(y)) \leq \Delta_{v}(x, y) \quad \text { for all } x, y \in \mathbb{P}^{1}(K) .
$$

In dynamical terminology, (4) says that $\varphi$ is $v$-adically nonexpanding. With our convention concerning congruences, the inequality (4) gives the following useful implication:

$$
\prod\left(\alpha_{i}-\beta_{i}\right) \equiv 0(\bmod \mathfrak{p}) \Rightarrow \prod\left(\varphi\left(\alpha_{i}\right)-\varphi\left(\beta_{i}\right)\right) \equiv 0(\bmod \mathfrak{p}) .
$$

Proof of Theorem 1. Without loss of generality, we may assume that the set $S$ contains all archimedean places of $K$ and all nonarchimedean places at which $\varphi$ has bad reduction. Let $\beta \in \mathbb{P}^{1}(K)$. If $\beta$ is preperiodic, then its orbit $\mathcal{O}_{\varphi}(\beta)$ is finite, so the orbit is discrete in $\mathbb{P}^{1}\left(K_{v}\right)$ for every place $v$. Hence in this case we have $\mathrm{C}\left(\mathcal{O}_{\varphi}(\beta)\right)=\mathcal{O}_{\varphi}(\beta)$, so the equality

$$
\mathcal{O}_{\varphi}(\beta) \cap Z(K)=\mathrm{C}\left(\mathcal{O}_{\varphi}(\beta)\right) \cap Z\left(\mathrm{~A}_{K}\right)
$$

is obvious.

We suppose now that $\beta \in \mathbb{P}^{1}(K)$ is a point with infinite $\varphi$-orbit. Since $Z$ is a finite set, there can be only finitely many iterates $\varphi^{n}(\beta)$ that lie in $Z$, so replacing $\beta$ with some $\varphi^{n}(\beta)$, we may assume that $\mathcal{O}_{\varphi}(\beta) \cap Z(K)=\emptyset$. Under this assumption, we suppose that there exists a point

$$
\alpha \in \mathrm{C}_{S}\left(\mathcal{O}_{\varphi}(\beta)\right) \cap Z\left(\mathrm{~A}_{K, S}^{\prime}\right)
$$

and derive a contradiction. The supposed existence of $\alpha$ means that there is an increasing sequence of positive integers

$$
\mathcal{N}=\left\{n_{1}, n_{2}, n_{3}, \ldots\right\}
$$


such that the sequence of points $\varphi^{n}(\beta)$ with $n \in \mathcal{N}$ converges adelically to $\alpha \in Z\left(\mathrm{~A}_{K, S}^{\prime}\right)$. Looking at each coordinate of the adele $\alpha$, we see in particular that for every prime $\mathfrak{p} \notin S$ of $K$ there exists an integer $N_{\mathfrak{p}}$ such that

$$
\varphi^{n}(\beta) \equiv \alpha_{\mathfrak{p}}(\bmod \mathfrak{p}) \quad \text { for all } n \in \mathcal{N} \text { with } n \geq N_{\mathfrak{p}} .
$$

Note that since $Z$ is a finite subset of $\mathbb{P}^{1}(K)$, each $\alpha_{\mathfrak{p}}$ is a point in $\mathbb{P}^{1}(K)$ considered as a subset of $\mathbb{P}^{1}\left(K_{\mathfrak{p}}\right)$.

We are going to use the dynamical Zsigmondy theorem from [4], whose statement we recall after making one definition.

Definition. Let $\varphi: \mathbb{P}^{1} \rightarrow \mathbb{P}^{1}$ and let $\gamma \in \mathbb{P}^{1}$. We say that $\varphi$ is of polynomial type at $\gamma$ if there is some $k \geq 1$ such that $\gamma$ is a totally ramified fixed point of $\varphi^{k}$.

REMARK 5. It is easy to check that $\varphi$ is of polynomial type at $\gamma$ if and only if, when we conjugate $\varphi$ by $(z-\gamma)^{-1}$, the map $\varphi^{k}$ is conjugated to a polynomial in $z$. We also remark that it is an exercise using the RiemannHurwitz genus formula to show that if $\varphi$ is of polynomial type at $\gamma$, then the $\varphi$-period of $\gamma$ is at most 2 ; cf. [11, Theorem 1.7].

Theorem 6 (Ingram-Silverman [4]). Let $K$ be a number field, let $S$ be a finite set of primes, let $\beta \in \mathbb{P}^{1}(K)$ be a point with infinite orbit, and let $\gamma \in \mathbb{P}^{1}(K)$ be a preperiodic point. Let $\varphi: \mathbb{P}^{1} \rightarrow \mathbb{P}^{1}$ be a $K$-morphism of degree at least two that is not of polynomial type at $\gamma$. There exists an integer $M=M_{\varphi, \beta, \gamma}$ so that for all $m \geq M$ there exists a prime ideal $\mathfrak{q}_{m} \notin S$ of $K$ satisfying

$$
\varphi^{m}(\beta) \equiv \gamma\left(\bmod \mathfrak{q}_{m}\right), \quad \varphi^{i}(\beta) \not \equiv \gamma\left(\bmod \mathfrak{q}_{m}\right) \quad \text { for all } 0 \leq i<m .
$$

In the terminology of [4], the prime ideal $\mathfrak{p}_{m}$ is a primitive divisor for $\varphi^{m}(\beta)-\gamma$. Theorem 6 says that $\varphi^{m}(\beta)-\gamma$ has a primitive divisor for all sufficiently large values of $m$. There are many classical theorems showing the existence of primitive divisors for the multiplicative group and for elliptic curves; see for example [7, 8, 12]. The proof of Theorem 6 uses a dynamical analog [10] of Siegel's theorem [9, IX.3.1] for integral points on elliptic curves.

REMARK 7. The theorem stated in [4] does not exclude a finite set of primes, but since $S$ is finite, we immediately get Theorem 6 from [4] by increasing $M$ so as to exclude the finitely many $m$ such that the primitive divisor of $\varphi^{m}(\beta)-\gamma$ is in $S$.

We fix an integer $R \geq 3$ and extend the field $K$ so that all of the periodic points of $\varphi$ of exact period $R$ are in $\mathbb{P}^{1}(K)$. Let $\gamma \in \mathbb{P}^{1}(K)$ be a point of exact period $R$ for $\varphi$. As noted in Remark 5 , the map $\varphi$ is not of polynomial 
type at $\gamma$, so we can apply Theorem 6 to find an integer $M=M_{\varphi, \beta, \gamma}$ such that $\varphi^{m}(\beta)-\gamma$ has a non- $S$ primitive divisor for all $m \geq M$. Doing this for each of the finitely many points of period $R$, we may choose one $M=M_{\varphi, \beta, R}$ that works for all $\gamma$ of period $R$.

Next we fix an integer $T \geq 1$ and we choose a non- $S$ primitive divisor for $\varphi^{m}(\beta)-\gamma$ for each $m$ between $M$ and $M+T$. Thus we find distinct prime ideals $\mathfrak{q}_{M, \gamma}, \mathfrak{q}_{M+1, \gamma}, \ldots, \mathfrak{q}_{M+T, \gamma} \notin S$ such that

$$
\varphi^{m}(\beta) \equiv \gamma\left(\bmod \mathfrak{q}_{m, \gamma}\right) \quad \text { for } M \leq m \leq M+T .
$$

We have indicated the dependence of $\mathfrak{q}_{m, \gamma}$ on both $m$ and $\gamma$, because we now replace $\gamma$ by $\varphi^{i} \gamma$ for each $1 \leq i<R$ and apply the same argument. Thus we find prime ideals $\mathfrak{q}_{m, \varphi^{i} \gamma} \notin S$ satisfying

$$
\varphi^{m}(\beta) \equiv \varphi^{i}(\gamma)\left(\bmod \mathfrak{q}_{m, \varphi^{i} \gamma}\right) \quad \text { for } M \leq m \leq M+T \text { and } 0 \leq i<R .
$$

Further, for any particular value of $i$, the ideals $\mathfrak{q}_{m, \varphi^{i} \gamma}$ are distinct for $M \leq m \leq M+T$. (These ideals also depend on $\varphi$ and $\beta$, of course, but $\varphi$ and $\beta$ are fixed throughout the proof.) This gives us a finite set of non- $S$ primes

$$
\mathcal{Q}=\mathcal{Q}_{R, T}=\left\{\mathfrak{q}_{m, \varphi^{i} \gamma}: M \leq m \leq M+T \text { and } 0 \leq i<R\right\} .
$$

(Note that $M=M_{\varphi, \beta, R}$ is independent of $T$.)

Consider the product

$$
\prod_{r=0}^{R-1}\left(\varphi^{m}(\beta)-\varphi^{r}(\gamma)\right) .
$$

It is divisible by each of the (not necessarily distinct) prime ideals

$$
\mathfrak{q}_{m, \gamma}, \mathfrak{q}_{m, \varphi \gamma}, \mathfrak{q}_{m, \varphi^{2} \gamma}, \ldots, \mathfrak{q}_{m, \varphi^{R-1} \gamma}
$$

(We remind the reader that according to our convention from Remark 3 , this "divisibility" is a brief way of saying that for each prime $\mathfrak{q}$ in the list (7), there is some $0 \leq r<R$ such that the two points $\varphi^{m}(\beta)$ and $\varphi^{r}(\gamma)$ reduce to the same point modulo $\mathfrak{q}$.)

We next show that the product

$$
\prod_{r=0}^{R-1}\left(\varphi^{n}(\beta)-\varphi^{r}(\gamma)\right)
$$

is divisible by all of the primes in the list (7) for every $n \geq m$. To see this, let $\mathfrak{q}$ be any prime listed in (7). We use the $R$-periodicity of $\gamma$, the assumption that $\varphi$ has good reduction at primes not in $S$, and Remark 4 to 
derive the implications

$$
\begin{aligned}
\prod_{r=0}^{R-1}\left(\varphi^{n}(\beta)-\varphi^{r}(\gamma)\right) \equiv 0(\bmod \mathfrak{q}) & \\
\Rightarrow & \prod_{r=0}^{R-1}\left(\varphi^{n+1}(\beta)-\varphi^{r+1}(\gamma)\right) \equiv 0(\bmod \mathfrak{q}) \quad \text { from }(5) \text { in Remark 4, } \\
\Rightarrow \prod_{r=0}^{R-1}\left(\varphi^{n+1}(\beta)-\varphi^{r}(\gamma)\right) \equiv 0(\bmod \mathfrak{q}) & \text { since } \gamma \text { is } R \text {-periodic. }
\end{aligned}
$$

Now an induction shows that for all $n \geq m$, the divisibility of (8) by the primes in the list (7) follows from the divisibility of (6).

Recall the sequence of positive integers $\mathcal{N}$ such that $\varphi^{n}(\beta)$ converges $\mathrm{A}_{K, S^{-}}^{\prime}$ adelically to $\alpha$ as $n \rightarrow \infty$ with $n \in \mathcal{N}$. In particular, for each $\mathfrak{q} \in \mathcal{Q}$ we have

$$
\varphi^{n}(\beta) \equiv \alpha_{\mathfrak{q}}(\bmod \mathfrak{q})
$$

for all sufficiently large $n \in \mathcal{N}$. (Note that $\mathcal{Q} \cap S=\emptyset$.) We recall that each $\alpha_{\mathfrak{q}}$ is chosen from the finite set $Z$, so we can reformulate (9) as

$$
\prod_{a \in Z}\left(\varphi^{n}(\beta)-a\right) \equiv 0(\bmod \mathfrak{q})
$$

for sufficiently large $n \in \mathcal{N}$ and for every $\mathfrak{q} \in \mathcal{Q}$.

For notational convenience, we define

$$
\mathfrak{Q}_{R, T}=\prod_{\mathfrak{q} \in \mathcal{Q}_{R, T}} \mathfrak{q}=\operatorname{Radical}\left(\prod_{M \leq m \leq M+T} \prod_{0 \leq i<R} \mathfrak{q}_{m, \varphi^{i} \gamma}\right) .
$$

The fact that the ideals $\mathfrak{q}_{m, \varphi^{i} \gamma}$ are distinct for fixed $i$ and varying $m$ implies that

$$
\# \mathcal{Q}_{R, T}>T,
$$

so $\mathfrak{Q}_{R, T}$ is a product of more than $T$ distinct prime ideals.

With this notation, we can rewrite (10) as

$$
\prod_{a \in Z}\left(\varphi^{n}(\beta)-a\right) \equiv 0\left(\bmod \mathfrak{Q}_{R, T}\right) \quad \text { for sufficiently large } n \in \mathcal{N} .
$$

Similarly, it follows from our earlier discussion that

$$
\prod_{r=0}^{R-1}\left(\varphi^{n}(\beta)-\varphi^{r}(\gamma)\right) \equiv 0\left(\bmod \mathfrak{Q}_{R, T}\right) \quad \text { for all } n \geq M_{R} .
$$


Comparing (11) and (12) for any single sufficiently large value of $n \in \mathcal{N}$, we conclude that

$$
\prod_{r=0}^{R-1} \prod_{a \in Z}\left(\varphi^{r}(\gamma)-a\right) \equiv 0\left(\bmod \mathfrak{Q}_{R, T}\right) .
$$

This last congruence is very interesting, because the product on the left-hand side does not depend on $T$, while the ideal $\mathfrak{Q}_{R, T}$ is a product of more than $T$ distinct prime ideals. Letting $T \rightarrow \infty$, we conclude that there is at least one value $r_{0}$ and at least one point $a_{0} \in Z$ such that

$$
\varphi^{r_{0}}(\gamma) \equiv a_{0}(\bmod \mathfrak{q})
$$

for infinitely many primes $\mathfrak{q}$. It follows that

$$
\varphi^{r_{0}}(\gamma)=a_{0} .
$$

To recapitulate, we have proven that given any point $\gamma \in \mathbb{P}^{1}$ that is periodic for $\varphi$ of period at least 3 , there is some point in the $\varphi$-orbit of $\gamma$ that lies in the set $Z$. But a rational map on $\mathbb{P}^{1}$ has periodic points of infinitely many periods. (More precisely, it has a periodic point of exact period $R$ for every $R \geq 5$; see [2, §6.8].) This contradicts the assumption that $Z$ is a finite set.

2. Local-global dynamics on $\mathbb{P}^{1}$ over function fields. In this section we prove the analogue of Theorem 1 for function fields. Let $k$ be an algebraically closed field and let $K / k$ be a function field, that is, $K$ is a finitely generated extension of $k$ of transcendence degree one. We employ the same notation as in the Introduction except that we consider only the places of $K / k$, i.e., the absolute values of $K$ that are trivial on $k$.

TheOREM 8. Let $K / k$ be a function field as above, let $S$ be a finite set of places of $K / k$, and let $\varphi: \mathbb{P}^{1} \rightarrow \mathbb{P}^{1}$ be a rational map of degree at least two defined over $K$. Let $Z \subset \mathbb{P}^{1}(K)$ be a finite set of points. Then with notation as in the Introduction,

$$
\mathcal{O}_{\varphi}(P) \cap Z(K)=\mathrm{C}_{S}\left(\mathcal{O}_{\varphi}(P)\right) \cap Z\left(\mathrm{~A}_{K, S}^{\prime}\right) .
$$

Proof. A rational map $\varphi$ defined over $K$ is isotrivial (or split) if there exists a Möbius transformation $M \in \mathrm{PGL}_{2}(\bar{K})$ such that $M \circ \varphi \circ M^{-1}$ has coefficients in $k$. As pointed out by T. Tucker [4, Remark 4], the Zsigmondy theorem in [4] can be proved for nonisotrivial rational maps over function fields using the results of Baker [1]. We observe that the characteristic zero assumption is not used in [1], and therefore is not needed to extend the results of [4], contrary to what is stated there. Thus the argument used to prove Theorem 1 applies verbatim in the function field case if $\varphi$ is nonisotrivial. 
Assume now that $\varphi$ is isotrivial. There is no loss of generality in extending $K$ by adjoining the coefficients of $M$, and then replacing $\varphi$ by $M \circ \varphi \circ M^{-1}$, we may assume that $\varphi$ has coefficients in $k$.

With an argument again as in the proof of Theorem 1, the case of preperiodic points is straightforward.

We now suppose that $\beta \in \mathbb{P}^{1}(K)$ is a point with infinite $\varphi$-orbit. Since $Z$ is a finite set, there can be only finitely many iterates $\varphi^{n}(\beta)$ that lie in $Z$, so replacing $\beta$ with some $\varphi^{n}(\beta)$, we may assume that $\mathcal{O}_{\varphi}(\beta) \cap Z=\emptyset$. Under this assumption, we suppose that there exists a point

$$
\alpha \in \mathrm{C}_{S}\left(\mathcal{O}_{\varphi}(\beta)\right) \cap Z\left(\mathrm{~A}_{K, S}^{\prime}\right)
$$

and derive a contradiction. The supposed existence of $\alpha$ means that there is an increasing sequence of positive integers

$$
\mathcal{N}=\left\{n_{1}, n_{2}, n_{3}, \ldots\right\}
$$

such that the sequence of points $\varphi^{n}(\beta)$ with $n \in \mathcal{N}$ converges adelically to $\alpha \in Z\left(\mathrm{~A}_{K, S}^{\prime}\right)$.

We note first that $\beta \notin k$, since otherwise the sequence $\varphi^{n_{i}}(\beta) \in k$ could not converge $\mathfrak{p}$-adically for any place $\mathfrak{p}$ of $K / k$ unless it became eventually constant, which would force $\beta$ to be preperiodic, contrary to our current assumption.

Assume now that $\varphi$ is not purely inseparable. Choose an element $b \in k$ that is periodic with respect to $\varphi$ and such that the backwards orbit of $b$ with respect to $\varphi$ is infinite and such that no element of $Z$ is in the (forward) orbit of $b$. It follows that the set $T$ of places $\mathfrak{p}$ of $K / k$ for which an element of $Z$ is congruent modulo $\mathfrak{p}$ to an element of the (forward) orbit of $b$ is finite. Now choose an element $a \in k$ in the backward orbit of $b$ with respect to $\varphi$, such that $a$ is not in the (forward) orbit of $b$ and such that the places of $K / k$ extending the place $\beta-a$ of $k(\beta)$ do not belong to $T$. Let $\mathfrak{p}$ be a place of $K / k$ extending the place $\beta-a$ of $k(\beta)$. By assumption, there exists $\alpha_{\mathfrak{p}} \in Z(K)$ with $\varphi^{n_{i}}(\beta)$ converging $\mathfrak{p}$-adically to $\alpha_{\mathfrak{p}}$. Thus, for all large $i$, we have

$$
\varphi^{n_{i}}(a) \equiv \varphi^{n_{i}}(\beta) \equiv \alpha_{\mathfrak{p}}(\bmod \mathfrak{p}) .
$$

But, for $i$ large, $\varphi^{n_{i}}(a)$ belongs to the forward orbit of $b$. This forces $\mathfrak{p} \in T$, which is a contradiction.

Finally, we have to deal with the case that $\varphi$ is purely inseparable. Without loss of generality, we may assume that $\varphi(z)=z^{q}$, where $q$ is a power of the characteristic of $K$. Let $a \in k$ and let $\mathfrak{p}$ be a place of $K / k$ extending the place $\beta-a$ of $k(\beta)$. Then the only way that a sequence $\varphi^{n_{i}}(\beta)$ can converge $\mathfrak{p}$-adically is if $a$ is in a finite field, and in this case it converges to an element in the Frobenius orbit of $a$. There are only finitely many such $a$ whose orbits intersect the finite set $Z$, and hence $\varphi^{n_{i}}(\beta)$ cannot converge adelically. This completes the proof. 


\section{Comments and speculations}

REMARK 9. We have stated our principal results (Theorems 1 and 8) for rational maps of degree at least two, but we can also consider maps of degree one. After a finite extension of the field $K$, any map $\varphi: \mathbb{P}^{1} \rightarrow \mathbb{P}^{1}$ of degree one may be conjugated to a map either of the form $\varphi(z)=a z$ or of the form $\varphi(z)=z+1$. There are three cases to consider.

(1) If $\varphi(z)=a z$ and $a$ is a root of unity, say $a^{n}=1$, then $\varphi^{n}(z)=z$ is the identity map, so every point has finite orbit. Hence the closure of $\mathcal{O}_{\varphi}(P)$ is equal to $\mathcal{O}_{\varphi}(P)$, so the conclusions of Theorems 1 and 8 are clearly true.

(2) If $\varphi(z)=a z$ and $a$ is not a root of unity, then we can mimic the proof of Theorem 1, replacing Theorem 6 with the classical Zsigmondy theorem for the multiplicative group. So Theorems 1 and 8 are also true in this case.

(3) If $\varphi(z)=z+1$, then Theorem 1 is not true in general. For example, take $Z=\{0, \infty\}$ and $\beta=1$. Then the orbit of $\beta$ is $\mathcal{O}_{\varphi}(\beta)=\{1,2,3, \ldots\}$, which does not intersect $Z$. On the other hand, the orbit of $\beta$ contains the subsequence

$$
\{n !: n=1,2,3, \ldots\},
$$

and this subsequence converges to the point in $Z\left(\mathrm{~A}_{\mathbb{Q}}\right)$ that is $\infty$ at the archimedean place and is 0 at all nonarchimedean places. Hence $\mathrm{C}\left(\mathcal{O}_{\varphi}(\beta)\right) \cap Z\left(\mathrm{~A}_{\mathbb{Q}}\right) \neq \emptyset$.

REMARK 10. Let $K / \mathbb{Q}$ be a number field, let $f(z) \in K(z)$ be a rational function of degree at least two, and let $\varphi(z)=z-f(z) / f^{\prime}(z)$. Iteration of $\varphi(z)$ is Newton's method of finding a root of $f(z)$. Let $\alpha \in K$ be a point whose orbit $\mathcal{O}_{\varphi}(\alpha)$ does not contain a root of $f$, e.g., any point that is not preperiodic for $\varphi$. Then Theorem 1 says that there are infinitely many places $v$ such that Newton's method applied to $\alpha$ does not converge to a root of $f(z)$. We raise the question of whether it is also true that there are infinitely many places such that Newton's method applied to $\alpha$ does converge to a root of $f(z)$. (It is not hard to show that this is the case for $\operatorname{deg}(f)=2$, but for maps of higher degree it is not clear.)

REMARK 11. We observe that an equality

$$
\mathcal{O}_{\varphi}(P) \cap Z(K)=\mathrm{C}\left(\mathcal{O}_{\varphi}(P)\right) \cap Z\left(\mathrm{~A}_{K}\right),
$$

such as given in [3] and in Theorem 1, provides an algorithm for determining whether $\mathcal{O}_{\varphi}(P) \cap Z(K)$ is nonempty. Thus "by day" one computes elements of the orbit and checks if they are in $Z$, while "by night" one checks if there are points in $\mathcal{O}_{\varphi}(P) \cap Z$ modulo $\mathfrak{Q}$ for ideals $\mathfrak{Q}$ that are more and more divisible by primes not in $S$. For a fixed $\mathfrak{Q}$ this is a finite computation. 
This is analogous to the fact that the Scharaschkin conjecture [6] gives an algorithm to decide whether a curve of genus at least two has any rational points (but not necessarily to find them all).

REMARK 12. The referee has asked whether Theorem 1 is true if the set $S$ is allowed to be an infinite set of places with density $\delta(S)=0$. This is an interesting question to which we do not know the answer.

Acknowledgements. The authors would like to thank the organizers of the TateFest (May 2008), at which event this research was initiated, and the referee for his helpful suggestions.

The first author's research was supported by NSF DMS-0650017.

\section{References}

[1] M. Baker, A finiteness theorem for canonical heights attached to rational maps over function fields, J. Reine Angew. Math. 626 (2009), 205-233.

[2] A. F. Beardon, Iteration of Rational Functions, Grad. Texts in Math. 132, Springer, New York, 1991.

[3] L.-C. Hsia and J. H. Silverman, On a dynamical Brauer-Manin obstruction, in: Proc. JA2007, J. Théor. Nombres Bordeaux, to appear.

[4] P. Ingram and J. H. Silverman, Primitive divisors in arithmetic dynamics, Math. Proc. Cambridge Philos. Soc. 146 (2009), 289-302.

[5] B. Poonen and J. F. Voloch, The Brauer-Manin obstruction for subvarieties of abelian varieties over function fields, Ann. of Math., to appear.

[6] V. Scharaschkin, Local-global problems and the Brauer-Manin obstruction, $\mathrm{PhD}$ thesis, Univ. of Michigan, 1999.

[7] A. Schinzel, Primitive divisors of the expression $A^{n}-B^{n}$ in algebraic number fields, J. Reine Angew. Math. 268/269 (1974), 27-33.

[8] J. H. Silverman, Wieferich's criterion and the abc-conjecture, J. Number Theory 30 (1988), 226-237.

[9] —, The Arithmetic of Elliptic Curves, 2nd ed., Grad. Texts in Math. 106, Springer, New York, 1992.

[10] -, Integer points, Diophantine approximation, and iteration of rational maps, Duke Math. J. 71 (1993), 793-829.

[11] —, The Arithmetic of Dynamical Systems, Grad. Texts in Math. 241, Springer, New York, 2007.

[12] K. Zsigmondy, Zur Theorie der Potenzreste, Monatsh. Math. Phys. 3 (1892), 265-284.

Mathematics Department

Brown University

Box 1917

151 Thayer Street

Providence, RI 02912, U.S.A.

E-mail: jhs@math.brown.edu
Department of Mathematics University of Texas

1 University Station C1200 Austin, TX 78712, U.S.A. E-mail: voloch@math.utexas.edu

Received on 16.6.2008

and in revised form on 24.10.2008 